\title{
Vinflunine treatment in patients with metastatic urothelial cancer: A Nordic retrospective multicenter analysis
}

\author{
KARIN HOLMSTEN $^{1}$, LINE HAMMER DOHN ${ }^{2}$, NIELS VIGGO JENSEN ${ }^{3}$, \\ CARL-HENRIK SHAH $^{1}$, FREDRIK JÄDERLING ${ }^{4}$, HELLE PAPPOT ${ }^{2}$ and ANDERS ULLÉN ${ }^{1}$ \\ ${ }^{1}$ Department of Oncology and Pathology, Karolinska University Hospital, SE-171 76 Stockholm, Sweden; \\ ${ }^{2}$ Department of Oncology, Rigshospitalet, 2100 Copenhagen; ${ }^{3}$ Department of Oncology, Odense University Hospital, \\ 5000 Odense, Denmark; ${ }^{4}$ Department of Radiology, Karolinska University Hospital, SE-171 76 Stockholm, Sweden
}

Received June 4, 2015; Accepted April 18, 2016

DOI: $10.3892 / 01.2016 .4775$

\begin{abstract}
In 2009, vinflunine was introduced as a second-line treatment to be used after the failure of platinum therapy in patients with metastatic urothelial carcinoma (mUC). The present study investigated the administered vinflunine to patients with mUC in standard clinical practice with the aim of evaluating treatment patterns, response, survival parameters and side-effects. Data were collected retrospectively from the first $100 \mathrm{mUC}$ patients treated with vinflunine at three Nordic cancer centers associated with the Nordic Urothelial Cancer Oncology Group. The overall response rate was $23 \%$ and complete response was observed in one patient. The median progression-free survival (mPFS) and median overall survival (mOS) were 2.8 (range, 0.5-34.3) and 6.3 (range, 0.3-39.7) months, respectively. An Eastern Cooperative Oncology Group performance status (ECOG PS) of 2 was present in $20 \%$ of the patients, and those patients exhibited significantly shorter mOS (4.1 vs. 7.0 months, $\mathrm{P}=0.001$ ) and a significantly higher degree of grade $3 / 4$ toxicity $(\mathrm{P}=0.026)$ compared with ECOG PS 0-1 patients. Furthermore, patients without visceral metastases had significantly longer mOS than patients with visceral metastases (10.6 vs. 6.0 months, $\mathrm{P}=0.008)$. The median number of cycles of vinflunine was 3 (range, 1-28). The current data confirms that vinflunine is an active agent for second-line treatment in an unselected clinical cohort of patients with mUC. ECOG PS and presence of visceral metastases were significant prognostic parameters. In particular, patients with ECOG PS 2 receiving vinflunine had a shorter mOS and a higher frequency of severe toxicity, and, thus, should be treated with caution. Furthermore, the present study observed large inter-individual differences in
\end{abstract}

Correspondence to: Dr Karin Holmsten, Department of Oncology and Pathology, Karolinska University Hospital, SE-171 76 Stockholm, Sweden

E-mail: karin.holmsten@ki.se

Key words: bladder cancer, platinum-refractory, vinflunine, prognostic factors radiological response and OS, indicating the need for further development of improved patient selection tools to optimize vinflunine treatment in platinum-refractory mUC patients.

\section{Introduction}

Metastatic urothelial carcinoma (mUC) is a chemotherapy-sensitive disease, and platinum-based chemotherapy is the standard primary treatment for patients with this form of cancer. Although the response rate to such treatment is $>50 \%$ (1), the vast majority of patients eventually develop platinum resistance and progressive disease, and are, thus, candidates for second-line treatment. During the recent decades, numerous therapeutic agents and combination regimens have been evaluated in the second-line setting of mUC treatment, including conventional cytotoxic agents (2-5) and targeted compounds (6-11). In general, these trials have been small and had negative outcomes, and very few treatments have been investigated in randomized trials.

In 2009, the European Medicines Agency approved vinflunine for second-line treatment of mUC based on the results of two phase II trials and one core randomized phase III trial (12). The phase III trial revealed that, compared with using best supportive care alone, the administration of vinflunine provided a small but significant median overall survival (mOS) benefit of 2.3 months in the eligible population $(\mathrm{P}=0.036)$. Despite the low response rate, disease control was achieved in $>40 \%$ of the patients with unaltered quality of life, and an acceptable and manageable toxicity profile (12). Based on these observations, vinflunine was the first compound to be approved for second-line treatment of mUC. Recently published long-term follow-up data confirmed the previously reported OS benefit (13) and vinflunine is the recommended second-line treatment in the European Association of Urology guidelines (14). This treatment strategy has been implemented in Denmark and Sweden.

The introduction of vinflunine should be considered a small but important step toward improved OS in patients with mUC (15). However, the treatment of platinum-refractory patients remains a clinical challenge, and further improvement in the outcome of such patients will require the development of novel and more efficient treatment strategies. 
Two potential approaches have emerged in this context: i) Using vinflunine as the backbone in a combined treatment strategy; and ii) identifying novel and more effective compounds. An example of the former strategy was recently reported by a German group that performed a phase I/II trial to evaluate the safety, tolerability, and efficacy of combining vinflunine with the antiangiogenic, multi-targeted receptor tyrosine kinase inhibitor, pazopanib (16). However, this combination was poorly tolerated, and the study was interrupted at dose level 1 due to toxicity. A similar phase I trial is currently being performed by the Nordic Urothelial Cancer Oncology Group (NUCOG) to analyze another kinase inhibitor, sorafenib, in combination with vinflunine (ClinicalTrials.gov Identifier: NCT01844947). Several other promising new drugs and approaches are also being investigated in phase I/II studies, including the following $(17,18)$ : Cytotoxic compounds (cabazitaxel, nab-paclitaxel); targeted drugs inhibiting mechanistic target of rapamycin (temsirolimus), phosphoinositide 3-kinase (buparlisib), human epidermal growth factor receptor $1 / 2$ (lapatinib) and fibroblast grow th factor receptor 3 (dovitinib); inhibitors of angiopoietin 1/2 (trebananib) and proteasomes (bortezomib); vaccine approaches (cdx-1307); and immunotherapy targeting the programmed cell death 1 signaling pathway.

Patients with platinum-refractory disease are heterogeneous in terms of performance status, comorbidities, renal function and the pattern of metastatic tumor burden, and, thus, they may benefit differently from second-line treatment. Bellmunt et al (19) demonstrated that the following three clinical parameters are independent prognostic factors in patients with platinum-refractory disease receiving second-line treatment with vinflunine: Low hemoglobin $(\mathrm{Hb})$ level $(<10 \mathrm{~g} / \mathrm{dl})$, an Eastern Cooperative Oncology Group performance status (ECOG PS) of $\geq 1$ and the presence of liver metastases. Based on these prognostic factors, the patients may be categorized into four groups with significant differences in mOS. Notably, the mOS was 14.2 months in patients with none of the adverse prognostic factors but was only 1.7 months in those with all three risk factors $(\mathrm{P}<0.001)(19)$. A recent retrospective analysis of seven prospective phase II trials demonstrated that short time from previous first-line chemotherapy is another risk factor significantly associated with poor prognosis. This finding may facilitate prognostic risk classification for second-line treatment (20), and has also led to the suggestion that nomograms can be used to predict the effect of second-line therapy (21). In further attempts to identify the patients that will most benefit from vinflunine treatment (22), it was demonstrated that a $>10 \%$ reduction in the sum of the longest axis diameter of the target lesions was a better early predictor of survival compared with response evaluation criteria in solid tumors (RECIST). Furthermore, it has been demonstrated that the type of platinum treatment administered prior to vinflunine (i.e., cisplatin or carboplatin) has no impact on the subsequent benefit of vinflunine $(20,23)$.

The overall aim of the present study was to evaluate the outcome and toxicity of second-line treatment with vinflunine administered to patients with mUC in standard clinical practice, and to identify the patients that most benefit from such treatment. The present study retrospectively analyzed collected demographic data, treatment patterns, survival parameters and side-effects for the first 100 patients treated with vinflunine at three Nordic cancer centers. The data were also assessed to determine whether they could be used to validate the three previously suggested prognostic factors for mUC: ECOG PS $\geq 1,<10 \mathrm{~g} / \mathrm{dl} \mathrm{Hb}$ and the presence of liver or visceral metastases $(19,24)$.

\section{Patients and methods}

Patients and data collection. The cohort comprised the first 100 mUC patients to be treated with vinflunine between February 2010 and July 2013 at three Nordic centers: Karolinska University Hospital (Stockholm, Sweden), Rigshospitalet (Copenhagen, Denmark) and Odense University Hospital (Odense, Denmark). All three of these centers participate in the NUCOG collaboration. The start of data collection coincided with the time points of the national decisions to initiate use of vinflunine as standard second-line treatment in the two countries (February 2010 in Denmark and July 2010 in Sweden).

Data were retrospectively collected from patient charts and covered the following: Patient and disease characteristics, prior treatment, including type of platinum-containing chemotherapy, vinflunine treatment, toxicity, response, and survival parameters. For further analyses, two subgroups were defined according to ECOG PS status: ECOG PS 0-1 and ECOG PS 2. Patients that had not received platinum-containing chemotherapy prior to vinflunine were excluded from the analyses of response and survival.

The trial was approved by the Research Ethics Committee in Stockholm, Sweden (approval no. Dnr 2013/664-31/3). Written informed consent from the patients was waived by the committee.

Definition of treatment response. Response rates were calculated based on standard radiological evaluations (i.e., computed or magnetic resonance tomography), conducted according to routine practice at each center. Responses were evaluated by the local radiologist at each center according to RECIST 1.1 criteria (25). The disease control rate (\%) was defined as the sum of patients with stable disease, partial response and complete response.

Definitions of PFS and OS. Progression-free survival (PFS) was defined as the period from the date of the first vinflunine treatment to the date of radiological progression. Patients with no radiological evaluation $(n=18)$ were excluded from the PFS analyses. OS was defined as the period from the date of the first vinflunine treatment to the date of mortality or the date of the most recent follow-up (26).

Toxicity. Toxicity assessment was graded according to the National Cancer Institute's Common Terminology Criteria for Adverse Events (NCI-CTCAE; version 4.0) (27). Grade 3 and 4 toxicities for all common vinflunine-associated hematological and non-hematological adverse events were recorded.

Statistical analysis. Univariate and multivariate analyses of time to event data (PFS and OS) were performed using the 
Table I. Characteristics of patients with metastatic uroethelial cancer.

\begin{tabular}{|c|c|c|c|}
\hline Characteristic & $\begin{array}{l}\text { All patients } \\
\quad(n=100)\end{array}$ & $\begin{array}{c}\text { ECOG PS 0-1 } \\
\quad(n=80)\end{array}$ & $\begin{array}{c}\text { ECOG PS } 2 \\
(n=20)\end{array}$ \\
\hline \multicolumn{4}{|l|}{ Gender } \\
\hline Male & $72(72)$ & $60(75)$ & $12(60)$ \\
\hline Female & $28(28)$ & $20(25)$ & $8(40)$ \\
\hline \multicolumn{4}{|l|}{ Age, years } \\
\hline Median & 68 & 68 & 68 \\
\hline Range & $45-80$ & $45-81$ & $46-79$ \\
\hline \multicolumn{4}{|l|}{ Primary tumor location } \\
\hline Bladder & $84(84)$ & $66(85)$ & $15(75)$ \\
\hline Upper urinary tract & $16(16)$ & $11(14)$ & $5(15)$ \\
\hline Urethra & $2(2)$ & $1(1)$ & $0(0)$ \\
\hline \multicolumn{4}{|l|}{ Metastatic site } \\
\hline Lymph nodes & $66(66)$ & $55(69)$ & $11(55)$ \\
\hline Lung & $48(48)$ & $35(44)$ & $13(65)$ \\
\hline Liver & $25(25)$ & $20(25)$ & $5(25)$ \\
\hline Bone & $18(18)$ & $14(18)$ & $4(20)$ \\
\hline Other & $17(17)$ & $14(18)$ & $3(15)$ \\
\hline \multicolumn{4}{|l|}{ Visceral metastases } \\
\hline No & $24(24)$ & $22(28)$ & $3(15)$ \\
\hline Yes & $76(76)$ & $58(72)$ & $17(85)$ \\
\hline \multicolumn{4}{|l|}{ ECOG PS } \\
\hline 0 & $30(30)$ & $30(38)$ & - \\
\hline 1 & $50(50)$ & $50(62)$ & - \\
\hline 2 & $20(20)$ & - & $20(100)$ \\
\hline \multicolumn{4}{|l|}{$\mathrm{GFR}, \mathrm{ml} / \mathrm{min}$} \\
\hline Median & 58 & 59 & 58 \\
\hline Range & $21-124$ & $27-124$ & $21-100$ \\
\hline$<10 \mathrm{~g} / \mathrm{dl} \mathrm{Hb}$ & $39(39)$ & $25(34)$ & $11(50)$ \\
\hline \multicolumn{4}{|l|}{ Primary curative treatment } \\
\hline Primary surgery & $40(77)$ & $33(77)$ & $7(78)$ \\
\hline Primary radiotherapy & $12(23)$ & $10(23)$ & $2(22)$ \\
\hline Perioperative chemotherapy & $14(27)$ & $10(23)$ & $4(44)$ \\
\hline Total & $52(52)$ & $43(54)$ & $9(45)$ \\
\hline \multicolumn{4}{|l|}{ Prior chemotherapy } \\
\hline Cisplatin/gemcitabine & $76(76)$ & $63(82)$ & $12(67)$ \\
\hline Carboplatin/gemcitabine & $17(17)$ & $14(18)$ & $4(22)$ \\
\hline Other & $2(2)$ & $0(0)$ & $2(11)$ \\
\hline \multicolumn{4}{|c|}{ Chemotherapy cycles prior to vinflunine, $\mathrm{n}$} \\
\hline Median & 6 & 6 & 6 \\
\hline Range & $0-20$ & $0-20$ & $0-16$ \\
\hline
\end{tabular}

All data, excluding age, GFR and cycles prior to vinflunine, is presented as n (\%). ECOG PS, Eastern Cooperative Oncology Group performance status; GFR, glomerular filtration rate; $\mathrm{Hb}$, hemoglobin.

log-rank (Mantel-Cox) model. Survival curves were estimated using the Kaplan-Meier method. Differences in nominal data were assessed by the Pearson $\chi^{2}$ test. $\mathrm{P}<0.05$ was used to indicate a statistically significant difference. Data were analyzed using SPSS statistics software for Windows (version 22.0; IBM SPSS, Armonk, NY, USA).

\section{Results}

Patient characteristics. Characteristics of the total patient cohort are listed in Table I. The cohort included $72 \%$ men and the median age was 68 years (range, $45-81$ years). The majority of the primary tumors $(84 \%)$ were located in the bladder and 
Table II. Vinflunine treatment, response and survival parameters.

\begin{tabular}{|c|c|c|c|}
\hline Parameter & $\begin{array}{l}\text { All patients } \\
\quad(n=100)\end{array}$ & $\begin{array}{l}\text { ECOG PS 0-1 } \\
\quad(n=80)\end{array}$ & $\begin{array}{c}\text { ECOG PS } 2 \\
(n=20)\end{array}$ \\
\hline \multicolumn{4}{|c|}{ Line of vinflunine therapy } \\
\hline First & $5(5)$ & $3(4)$ & $2(10)$ \\
\hline Second & $94(94)$ & $76(95)$ & $18(90)$ \\
\hline Third & $1(1)$ & $1(1)$ & $0(0)$ \\
\hline \multicolumn{4}{|l|}{ Cycles, n } \\
\hline Median & 3 & 4 & 2 \\
\hline Range & $1-28$ & $1-18$ & $1-21$ \\
\hline \multicolumn{4}{|c|}{ Initial dose, $\mathrm{mg} / \mathrm{m}^{2}$} \\
\hline 320 & $33(35)$ & $31(41)$ & $2(10)$ \\
\hline 280 & $44(46)$ & $34(45)$ & $10(53)$ \\
\hline 250 & $18(19)$ & $11(14)$ & $7(37)$ \\
\hline \multicolumn{4}{|l|}{ Response $^{\mathrm{a}}$} \\
\hline $\mathrm{CR}$ & $1(1)$ & $1(2)$ & $0(0)$ \\
\hline PR & $17(22)$ & $17(27)$ & $0(0)$ \\
\hline SD & $25(32)$ & $22(35)$ & $3(20)$ \\
\hline PD & $34(44)$ & $22(35)$ & $12(80)$ \\
\hline \multicolumn{4}{|c|}{ mPFS, months } \\
\hline Median & 2.8 & 2.8 & 1.8 \\
\hline Range & $0.5-34.3$ & $0.7-34.3$ & $1.4-14.7$ \\
\hline \multicolumn{4}{|c|}{ mOS, months } \\
\hline Median & 6.3 & 7.0 & 4.1 \\
\hline Range & $0.3-39.7$ & $0.3-39.7$ & $1.2-16.3$ \\
\hline
\end{tabular}

All data, excluding cycles, mPFS and mOS, are presented as n (\%). ${ }^{\text {an }}$ total, 18 patients did not undergo radiological evaluation. ECOG PS, Eastern Cooperative Oncology Group performance status; CR, complete response; PR, partial response; SD, stable disease; PD, progressive disease; mPFS, median progression-free survival; mOS, median overall survival.

approximately half (52\%) of the patients had undergone primary curative treatment with surgery (cystectomy or nephroureterectomy) or radiotherapy. Furthermore, $27 \%$ of those administered with primary curative treatment had received perioperative chemotherapy with three cycles of gemcitabine and cisplatin.

The vast majority (95\%) of the patients had received first-line platinum-containing chemotherapy (cisplatin or carboplatin) prior to vinflunine. Five patients underwent no chemotherapy prior to vinflunine and were excluded from further analyses of response and survival. A median of 6 cycles of first-line chemotherapy (range, 0-20 cycles) was administered prior the commencement of vinflunine treatment (Table I), and $62 \%$ of the patients received $\geq 6$ cycles. Grounds for discontinuing first-line treatment were as follows: Disease progression (88\%), toxicity (9\%) and other reasons (3\%; data not shown).

At the onset of vinflunine treatment, $4 \%$ of patients had local recurrence in the bladder and $96 \%$ had metastatic disease. The most common locations of metastases were lymph nodes (66\%), lungs (48\%) and the liver (25\%). No evidence of visceral metastases was observed in $24 \%$ of the patients. The ECOG PS was 0 in $30 \%, 1$ in $50 \%$ and 2 in $20 \%$ of the patients (Table I).

Vinflunine treatment. Vinflunine was administered as first-line treatment in $5 \%$ of patients, as second-line treatment in $94 \%$ and as third-line treatment in $1 \%$ (Table II). The median number of cycles of vinflunine treatment was 3 (range, 1-28) and the starting dose was $280 \mathrm{mg} / \mathrm{m}^{2}$ in $46 \%$ of the patients. Dose reduction was performed in $19 \%$ and treatment delay occurred in $18 \%$. In addition, $4 \%$ of patients underwent re-challenge with vinflunine and $8 \%$ received different chemotherapy after vinflunine, primarily taxane-based combination chemotherapy.

Toxicity. Grade 3 and 4 toxicities, according to NCI-CTCAE version 4.0 , were recorded in a total of $76 \%$ of patients treated with vinflunine. The specific adverse events are listed in Table III. The proportion of patients with $\geq 1$ grade $3 / 4$ toxicity was significantly higher in those with ECOG PS 2 than in those with ECOG PS 0-1 (95 vs. 71\%, respectively; $\mathrm{P}=0.026$ ). The patients with ECOG PS 2 also exhibited a significantly higher frequency of fatigue compared with ECOG PS 0-1 patients $(\mathrm{P}=0.012)$.

Response. The overall response rate to second-line vinflunine chemotherapy was $23 \% ; 1 \%$ of patients exhibited a complete response (CR) and 22\% exhibited a partial response (PR). The disease control rate (DCR) was $55 \%$ (Fig. 1). For patients with ECOG PS 0-1, 2\% exhibited CR, 27\% PR and 64\% DCR. No 
Table III. Safety according to grade $3 / 4$ toxicity ${ }^{\mathrm{a}}$.

\begin{tabular}{|c|c|c|c|c|}
\hline Toxicity & $\begin{array}{l}\text { All patients } \\
\quad(n=100)\end{array}$ & $\begin{array}{c}\text { ECOG PS 0-1 } \\
\quad(n=80)\end{array}$ & $\begin{array}{c}\text { ECOG PS } 2 \\
\quad(n=20)\end{array}$ & P-value \\
\hline \multicolumn{5}{|l|}{ Hematological } \\
\hline Anemia & $33(33)$ & $23(29)$ & $10(50)$ & 0.071 \\
\hline Neutropenia & $23(23)$ & $19(24)$ & $4(20)$ & 0.722 \\
\hline Febrile neutropenia & $13(13)$ & $10(13)$ & $3(15)$ & 0.766 \\
\hline Thrombocytopenia & $3(3)$ & $3(4)$ & $0(0)$ & 0.379 \\
\hline \multicolumn{5}{|l|}{ Non-hematological } \\
\hline Fatigue & $36(36)$ & $24(30)$ & $12(60)$ & 0.012 \\
\hline Constipation & $22(22)$ & $20(25)$ & $2(10)$ & 0.148 \\
\hline Abdominal pain & $12(12)$ & $8(10)$ & $4(20)$ & 0.218 \\
\hline Infusion site reaction & $5(5)$ & $4(5)$ & $1(5)$ & 1.000 \\
\hline Stomatitis & $4(4)$ & $4(5)$ & $0(0)$ & 0.518 \\
\hline Nausea & $2(2)$ & $2(3)$ & $0(0)$ & 0.475 \\
\hline Vomiting & $2(2)$ & $2(3)$ & $0(0)$ & 0.475 \\
\hline Myalgia & $2(2)$ & $2(3)$ & $0(0)$ & 0.475 \\
\hline$\geq 1$ Toxicity & $76(76)$ & $57(71)$ & $19(95)$ & 0.026 \\
\hline
\end{tabular}

All data are presented as $\mathrm{n}(\%) .{ }^{\mathrm{a}}$ Graded according to the National Cancer Institute's Common Terminology Criteria for Adverse Events (version 4.0). P-value showing the difference between ECOG PS 0-1 and 2 was assessed by the Pearson's $\chi^{2}$ test. ECOG PS, Eastern Cooperative Oncology Group performance status.

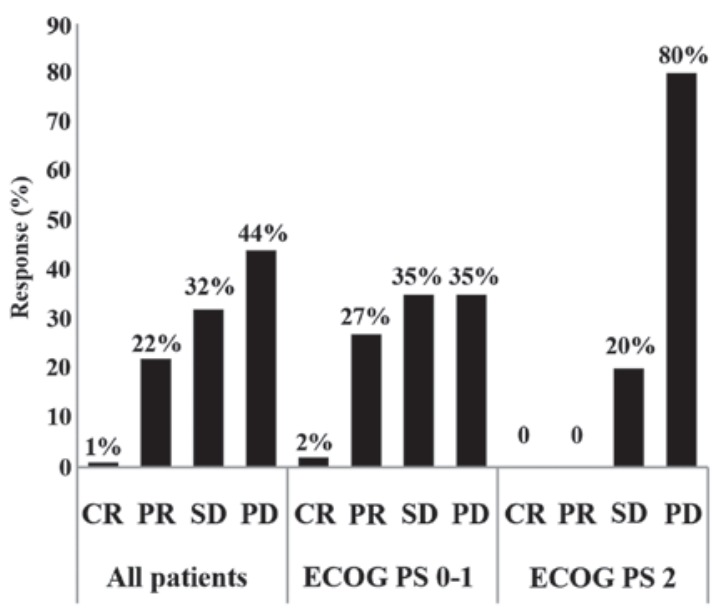

Figure 1. Comparison of response to vinflunine treatment in patients with mUC subdivided into three groups. CR, complete response; PR, partial response; SD, stable disease; PD, progressive disease; ECOG PS, Eastern Cooperative Oncology Group performance status.

responses were noted for patients with ECOG PS 2; instead, $20 \%$ of the patients presented stable disease (SD) as best response. In the whole study population, patients presenting CR or PR had significantly longer mOS compared with those with SD or progressive disease (PD) (13.8 vs. 6.1 months, respectively; $\mathrm{P}=0.028$ ). In the ECOG PS $0-1$ and 2 groups, there were no significant difference in mOS between patients with $\mathrm{CR}$ or PR and those with SD or PD (data not shown).

Survival data. The mPFS and mOS were 2.8 (range, 0.5-34.3) and 6.3 (range, 0.3-39.7) months, respectively (Table II;
Fig. 2A and B). Patients with ECOG PS 2 had short mPFS and mOS (1.8 and 4.1 months, respectively). Among patients with ECOG PS $0-1$, OS was $>12$ months in $21 \%$ and $>24$ months in $5 \%$. The corresponding proportions for patients with ECOG PS 2 were 10 and $0 \%$, respectively. No significant difference in OS in association with the presence or absence of $\geq 1$ grade $3 / 4$ toxicity was noted in either of the cohorts (data not shown).

Prognostic factors. Performance status was strongly associated with OS. The mOS was significantly shorter for patients with ECOG PS 2 compared with those with ECOG PS 0-1 [4.1 (range 1.2-16.3) vs. 7.0 (range 0.3-39.7) months, $\mathrm{P}=0.001$; Fig. 3A]. Furthermore, the mOS was significantly longer for patients without visceral metastases at onset of vinflunine treatment $(22 \%)$ compared with patients with such metastases at that time [10.6 (range 1.6-39.7) vs. 6.0 (range 0.3-28.1) months, $\mathrm{P}=0.008$; Fig. $3 \mathrm{~B}]$. The mOS were 18.1 months for patients with ECOG PS 0-1 and no visceral metastases (20\%). Presence of liver metastasis or $\mathrm{Hb}<10 \mathrm{~g} / \mathrm{dl}$ was not significantly correlated with OS. Also, none of the following parameters showed significant correlations with OS: Alkaline phosphatase, the initial dose of vinflunine, age of $>65$ years or presence of grade $3 / 4$ toxicity.

The number of cycles of chemotherapy administered prior to the onset of vinflunine was significantly correlated with OS $(\mathrm{P}=0.016)$, in favor for patients who had previously received $\geq 6$ cycles of platinum-containing chemotherapy compared with those administered <6 cycles (6.9 vs. 4.7 months). Such a correlation was also observed in the group with ECOG PS 0-1 $(\mathrm{P}=0.029)$, but not in the group with ECOG PS $2(\mathrm{P}=0.480)$. 

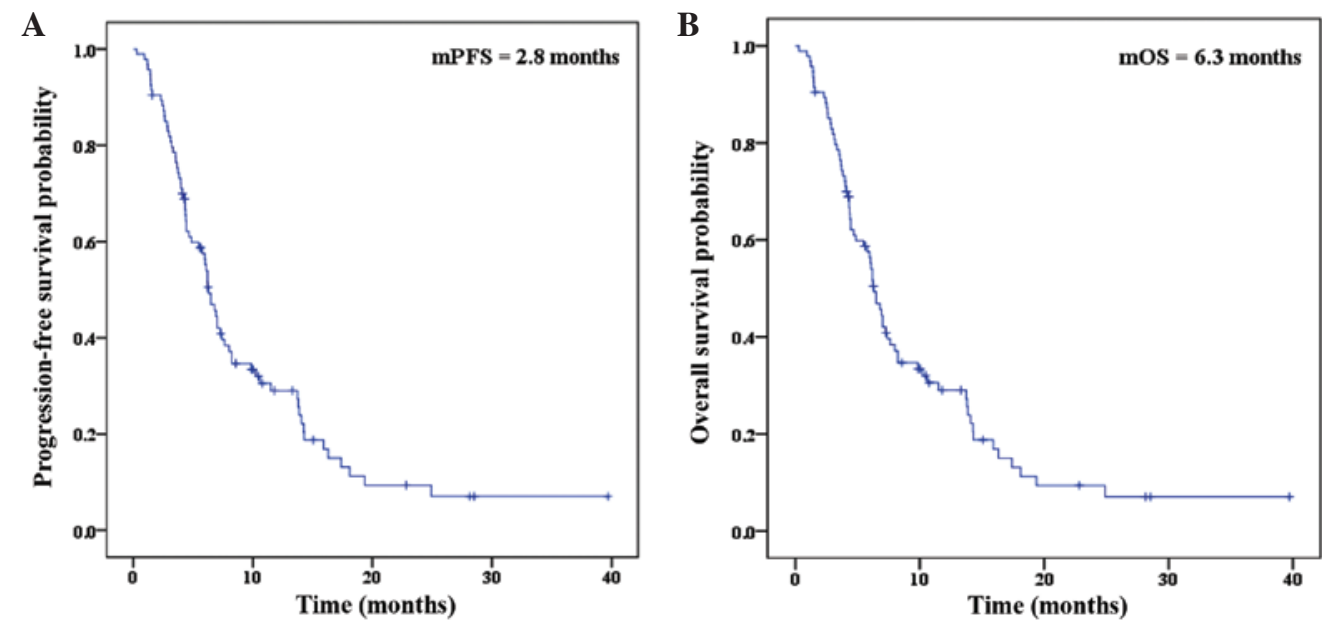

Figure 2. Survival data for patients with metastatic urothelial carcinoma treated with vinflunine as second-line chemotherapy, illustrated by Kaplan-Meier curves. (A) mPFS of 2.8 months (range, 0.5-34.3 months) and (B) mOS of 6.3 months (range, 0.3-39.7 months). mPFS, median progression-free survival; mOS, median overall survival.
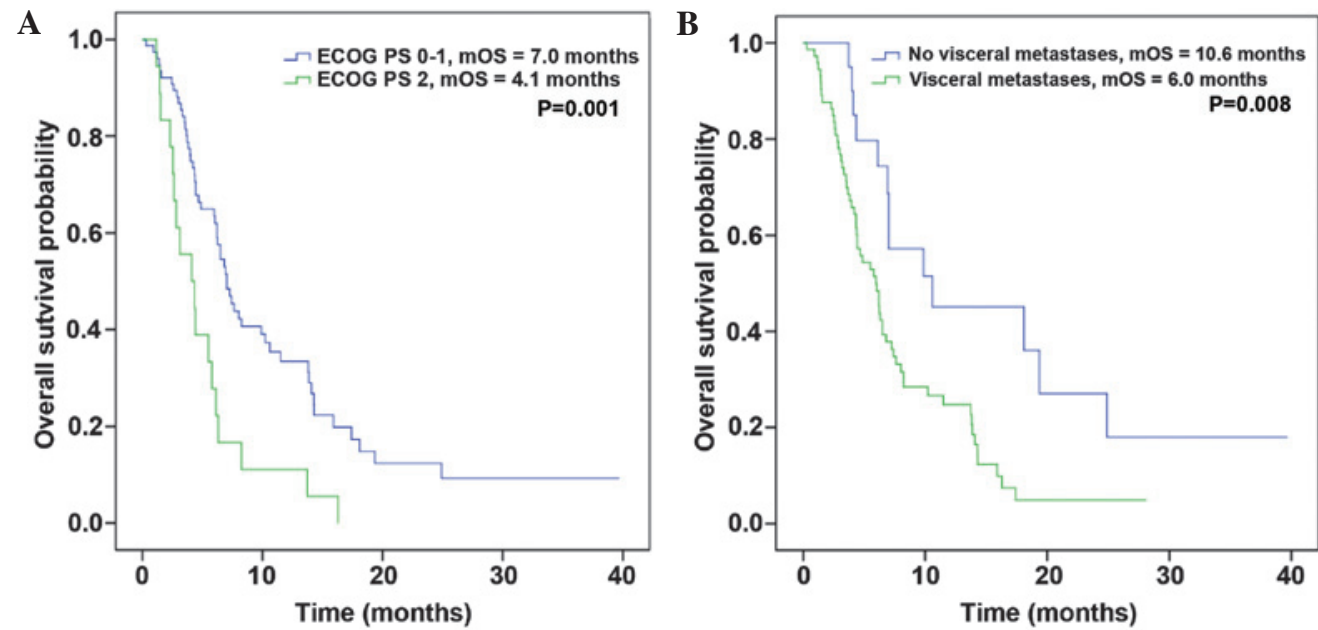

Figure 3. Kaplan-Meier curves illustrating mOS data for patients with metastatic urothelial carcinoma treated with vinflunine as second-line chemotherapy in relation to different prognostic factors. (A) OS as a function of ECOG PS 0-1 vs. ECOG PS 2 (mOS: 7.0 vs. 4.1 months; P=0.001). (B) OS as a function of absence vs. presence of visceral metastases (mOS: 10.6 vs. 6.0 months; $\mathrm{P}=0.008$ ). ECOG PS, Eastern Cooperative Oncology Group performance status; mOS, median overall survival.

\section{Discussion}

Platinum-refractory mUC is a medical challenge due to the aggressiveness of the disease and the heterogeneity of the patient population. Certain patients with mUC present with slowly progressing limited disease and good performance status, whereas others may present with large-volume disease that affects vital organs, impaired renal function, significant co-morbidity and poor overall performance status. Furthermore, the inter-individual sensitivity to chemotherapy differs, and represents an additional factor that is uncontrollable and influences the benefits of the treatment administered.

No standard second-line chemotherapy for mUC was previously available, however, in 2009, the use of vinflunine was approved based on positive results of a randomized phase III trial demonstrating its beneficial effects on OS (12). Furthermore, the overall response rate, disease control rate and PFS for vinflunine all significantly favored treating patients with this drug as opposed to providing best supportive care only, thus strengthening the rationale for offering vinflunine to platinum-refractory patients. The European Medical Agency has approved vinflunine as a second-line treatment and the compound is recommended with the highest level of evidence in the European Association of Urology guidelines (14). In the current study, the first 100 patients treated with vinflunine at three high-volume cancer centers affiliated with NUCOG were retrospectively analyzed. The overall aim was to evaluate treatment patterns, efficacy data and side effects of vinflunine in a real-world clinical setting, and also to relate those aspects to previously demonstrated prognostic factors for mUC in patients treated with second-line chemotherapy.

It was identified that the patients included in the present study were heterogeneous with regard to the line of vinflunine treatment and ECOG PS. In addition, these patients differed from those included in the phase III trial conducted by Bellmunt et al (12), all of whom were treated with second-line vinflunine therapy and had an ECOG PS of $<2$. More specifically, $20 \%$ of the patients in the current study presented with 
ECOG PS 2 at onset of treatment, and $5 \%$ of the patients were treated with first-line and $1 \%$ with third-line vinflunine therapy. ECOG PS is well known to predict the benefit of subsequent chemotherapy in mUC patients $(19,24)$; therefore, two sub-cohorts were identified for further comparison: Patients with ECOG PS 0-1 and patients with ECOG PS 2.

The mPFS and mOS were marginally shorter in the present study (2.8 and 6.3 months) compared with the phase III trial (3.0 and 6.9 months) conducted by Bellmunt et al (12), possibly because survival data were calculated from the first day of vinflunine treatment in the current investigation but from the randomization date in the previous trial. Patients with ECOG PS 2 had significantly shorter mOS (4.1 months) compared with those with ECOG PS 0-1 (7.0 months), and they also had higher rates of grades 3 and 4 toxicity. The present study is the first to evaluate ECOG PS 2 patients treated with vinflunine and the results demonstrate that, in general, vinflunine should be used with caution in such patients. This finding agrees with the conclusions that De Santis et al (28) reported after evaluating chemotherapy in a cisplatin-unfit population, including ECOG PS 2 patients.

The patients in the current cohort underwent a median of 3 cycles of vinflunine, which is similar to treatment with this drug in the trial by Bellmunt et al (12). However, the inter-individual variation in the number of vinflunine cycles was large (range, 1-28 cycles) in the present study, which indicates significant differences in treatment benefit. Re-exposure to vinflunine in a second course of treatment was performed in $4 \%$ of the patients that exhibited clinical benefit after the first series of vinflunine treatment; these patients were entered into surveillance but eventually progressed. Future studies should explore this re-exposure approach in selected patients showing a significant clinical benefit after the first series of vinflunine treatment. A considerable proportion of the vinflunine-treated patients in the phase III trial were identified as long-term survivors: $27 \%$ with OS $>12$ months and $11 \%$ with OS > 24 months (13). Similarly, in the current trial, $23 \%$ had an OS $>12$ months and $5 \%$ had an OS of $>24$ months. It is possible that such patients harbor tumors with an extraordinary vinflunine-sensitive genotype, however, no biomarkers from patient tumor samples or other tissues have yet been identified as predictive of the efficacy of vinflunine treatment.

The patients without visceral metastases at the time of initiation of vinflunine had a significantly longer mOS compared with patients with such metastases at that time (10.6 vs. 6.0 months, $\mathrm{P}=0.008$ ), supporting previous observations that this parameter is of high prognostic value $(17,23)$. In the present study, mOS was significantly shorter for patients with ECOG PS 2 than for those with ECOG PS 0-1 $(\mathrm{P}=0.001)$. Furthermore, the presence of liver metastases and $<10 \mathrm{~g} / \mathrm{dl} \mathrm{Hb}$ were not of prognostic value, possibly due to the heterogeneity in vinflunine treatment and performance status (ECOG PS 0-2).

In summary, to the best of our knowledge, the present study represents the largest retrospective analysis conducted to date to focus on advanced mUC treated with vinflunine, and the results confirmed the activity of this drug in an unselected cohort of patients treated in routine clinical practice. The median PFS and OS values observed are similar to data obtained in a previous randomized phase III trial. The patients with ECOG PS 2 had a shorter mOS and a higher frequency of severe toxicity; hence, such individuals should be treated with caution. ECOG PS $>1$ and the presence of visceral metastases were confirmed as significantly adverse prognostic parameters that should be taken into consideration when prescribing vinflunine treatment. Notably, large inter-individual differences were identified regarding OS and radiological response. The aforementioned observations call for the development of improved patient selection tools that should preferably include tumor biomarkers relevant for determining the sensitivity to vinflunine treatment.

\section{Acknowledgements}

The present study was supported by grants awarded to Dr Anders Ullén from the Swedish Cancer Society (grant no. 130277) and the Stockholm Cancer Society (grant nos. 141433 and 144233).

\section{References}

1. von der Maase H, Hansen SW, Roberts JT, Dogliotti L, Oliver T, Moore MJ, Bodrogi I, Albers P, Knuth A, Lippert CM, et al: Gemcitabine and cisplatin versus methotrexate, vinblastine, doxorubicin, and cisplatin in advanced or metastatic bladder cancer: Results of a large, randomized, multinational, multicenter, phase III study. J Clin Oncol 18: 3068-3077, 2000.

2. Lorusso V, Pollera CF, Antimi M, Luporini G, Gridelli C, Frassineti GL, Oliva C, Pacini M and De Lena M: A phase II study of gemcitabine in patients with transitional cell carcinoma of the urinary tract previously treated with platinum. Italian Co-operative Group on Bladder Cancer. Eur J Cancer 34: 1208-1212, 1998

3. McCaffrey JA, Hilton S, Mazumdar M, Sadan S, Kelly WK Scher HI and Bajorin DF: Phase II trial of docetaxel in patients with advanced or metastatic transitional-cell carcinoma. J Clin Oncol 15: 1853-1857, 1997.

4. Roth BJ, Manola J, Dreicer R, Graham D and Wilding G; Eastern Cooperative Oncology Group: Piritrexim in advanced, refractory carcinoma of the urothelium (E3896): A phase II trial of the Eastern Cooperative Oncology Group. Invest New Drugs 20: 425-429, 2002.

5. Witte RS, Manola J, Burch PA, Kuzel T, Weinshel EL and Loehrer PJ Sr: Topotecan in previously treated advanced urothelial carcinoma: An ECOG phase II trial. Invest New Drugs 16: 191-195, 1998.

6. Choueiri TK, Ross RW, Jacobus S, Vaishampayan U, Yu EY, Quinn DI, Hahn NM, Hutson TE, Sonpavde G, Morrissey SC, et al: Double-blind, randomized trial of docetaxel plus vandetanib versus docetaxel plus placebo in platinum-pretreated metastatic urothelial cancer. J Clin Oncol 30: 507-512, 2012.

7. Gallagher DJ, Milowsky MI, Gerst SR, Ishill N, Riches J, Regazzi A, Boyle MG, Trout A, Flaherty AM and Bajorin DF: Phase II study of sunitinib in patients with metastatic urothelial cancer. J Clin Oncol 28: 1373-1379, 2010.

8. Gerullis H, Eimer C, Ecke TH, Georgas E, Freitas C, Kastenholz S, Arndt C, Heusch C and Otto T: A phase II trial of temsirolimus in second-line metastatic urothelial cancer. Med Oncol 29: 2870-2876, 2012.

9. Pili R, Qin R, Flynn PJ, Picus J, Millward M, Ho WM, Pitot H, Tan W, Miles KM, Erlichman C and Vaishampayan U: A phase II safety and efficacy study of the vascular endothelial growth factor receptor tyrosine kinase inhibitor pazopanib in patients with metastatic urothelial cancer. Clin Genitourin Cancer 11: 477-483, 2013.

10. Seront E, Rottey S, Sautois B, Kerger J, D'Hondt LA, Verschaeve V, Canon JL, Dopchie C, Vandenbulcke JM, Whenham N, et al: Phase II study of everolimus in patients with locally advanced or metastatic transitional cell carcinoma of the urothelial tract: Clinical activity, molecular response, and biomarkers. Ann Oncol 23: 2663-2670, 2012. 
11. Wülfing C, Machiels JP, Richel DJ, Grimm MO, Treiber U, De Groot MR, Beuzeboc P, Parikh R, Pétavy F and El-Hariry IA: A single-arm, multicenter, open-label phase 2 study of lapatinib as the second-line treatment of patients with locally advanced or metastatic transitional cell carcinoma. Cancer 115: 2881-2890, 2009.

12. Bellmunt J, Théodore C, Demkov T, Komyakov B, Sengelov L, Daugaard G, Caty A, Carles J, Jagiello-Gruszfeld A, Karyakin O, et al: Phase III trial of vinflunine plus best supportive care compared with best supportive care alone after a platinum-containing regimen in patients with advanced transitional cell carcinoma of the urothelial tract. J Clin Oncol 27: 4454-4461, 2009.

13. Bellmunt J, Fougeray R, Rosenberg JE, von der Maase H, Schutz FA, Salhi Y, Culine S and Choueiri TK: Long-term survival results of a randomized phase III trial of vinflunine plus best supportive care versus best supportive care alone in advanced urothelial carcinoma patients after failure of platinum-based chemotherapy. Ann Oncol 24: 1466-1472, 2013.

14. Witjes JA, Compérat E, Cowan NC, De Santis M, Gakis G, Lebrét T, Ribal MJ, van der Heijden AG and Sherif A; European Association of Urology: EAU guidlines on muscle-invasive and metastatic bladder cancer: Summary of the 2013 guidelines. Eur Urol 65: 778-792, 2014.

15. Razzak M: Urothelial carcinoma: Vinflunine offers modest but important survival benefit. Nat Rev Urol 10: 186, 2013.

16. Gerullis H, Eimer C, Ecke TH, Georgas E, Arndt C and Otto T: Combined treatment with pazopanib and vinflunine in patients with advanced urothelial carcinoma refractory after first-line therapy. Anticancer Drugs 24: 422-425, 2013.

17. Clinical Trials.gov: Phase I Study With Sorafenib in Addition to Vinflunine in Metastatic Transitional Cell Carcinoma of the Urothelial Tract (VINSOR). https://clinicaltrials.gov/ct2/show/NCT01844947. Accessed March 17, 2014.

18. Sonpavde G, Jones BS, Bellmunt J, Choueiri TK and Sternberg CN: Future directions and targeted therapies in bladder cancer. Hematol Oncol Clin North Am 29: 361-376, 2015

19. Bellmunt J, Choueiri TK, Fougeray R, Schutz FA, Salhi Y, Winquist E, Culine S, von der Maase H, Vaughn DJ and Rosenberg JE: Prognostic factors in patients with advanced transitional cell carcinoma of the urothelial tract experiencing treatment failure with platinum-containing regimens. J Clin Oncol 28: 1850-1855, 2010.
20. Sonpavde G, Pond GR, Fougeray R, Choueiri TK, Qu AQ Vaughn DJ, Niegisch G, Albers P, James ND, Wong YN, et al: Time from prior chemotherapy enhances prognostic risk grouping in the second-line setting of advanced urothelial carcinoma: A retrospective analysis of pooled, prospective phase 2 trials. Eur Urol 63: 717-723, 2013.

21. Pond GR, Agarwal N, Bellmunt J, Choueiri TK, Qu A, Fougeray R, Vaughn D, James ND, Salhi Y, Albers P, et al: A nomogram including baseline prognostic factors to estimate the activity of second-line therapy for advanced urothelial carcinoma. BJU Int 113: E137-E143, 2014.

22. Krajewski KM, Fougeray R, Bellmunt J, Pons F, Schutz FA, Rosenberg JE, Salhi Y and Choueiri TK: Optimisation of the size variation threshold for imaging evaluation of response in patients with platinum-refractory advanced transitional cell carcinoma of the urothelium treated with vinflunine. Eur J Cancer 48: 1495-1502, 2012.

23. Pond GR, Bellmunt J, Fougeray R, Choueiri TK, Qu AQ, Niegisch G, Albers P, Di Lorenzo G, Salhi Y, Galsky MD, et al: Impact of response to prior chemotherapy in patients with advanced urothelial carcinoma receiving second-line therapy: Implications for trial design. Clin Genitourin Cancer 11: 495-500, 2013.

24. Bajorin DF, Dodd PM, Mazumdar M, Fazzari M, McCaffrey JA Scher HI, Herr H, Higgins G and Boyle MG: Long-term survival in metastatic transitional-cell carcinoma and prognostic factors predicting outcome of therapy. J Clin Oncol 17: 3173-3181, 1999.

25. Eisenhauer EA, Therasse P, Bogaerts J, Schwartz LH, Sargent D, Ford R, Dancey J, Arbuck S, Gwyther S, Mooney M, et al: New response evaluation criteria in solid tumours: Revised RECIST guideline (version 1.1). Eur J Cancer 45: 228-247, 2009.

26. Punt CJ, Buyse M, Köhne CH, Hohenberger P, Labianca R, Schmoll HJ, Påhlman L, Sobrero A and Douillard JY: Endpoints in adjuvant treatment trials: A systematic review of the literature in colon cancer and proposed definitions for future trials. J Natl Cancer Inst 99: 998-1003, 2007.

27. U.S. Department of Health and Human Services: National Institutes of Health, National Cancer Institute: Common Terminology Criteria for Adverse Events (CTCAE). Version 4.0, 2009.

28. De Santis M, Bellmunt J, Mead G, Kerst JM, Leahy M, Maroto P Gil T, Marreaud S, Daugaard G, Skoneczna I, et al: Randomized phase II/III trial assessing gemcitabine/carboplatin and methotrexate/carboplatin/vinblastine in patients with advanced urothelial cancer who are unfit for cisplatin-based chemotherapy: EORTC study 30986. J Clin Oncol 30: 191-199, 2012. 